\title{
Surgical Treatment of Gallstone Ileus: Less Is More
}

\author{
Cristina Vera-Mansilla $^{a}$ Ana Sanchez-Gollarte ${ }^{a}$ Belen Matias $^{a}$ \\ Fernando Mendoza-Moreno $^{a}$ Manuel Díez-Alonso $^{a}$ \\ Francisca Garcia-Moreno Nisa ${ }^{a, b}$ \\ aGeneral Surgery Department, Hospital Universitario Príncipe de Asturias, Alcala de Henares, Spain; \\ bDepartment of Surgery and Medical and Social Sciences, GIBBYC-UAH CIBER-BBN IRYCIS, Universidad de Alcalá, \\ Alcala de Henares, Spain
}

\section{Keywords}

Gallstone ileus · Enterolithotomy · Intestinal obstruction ·

Bouveret syndrome

\begin{abstract}
Introduction: The objective of this study was to evaluate the need for cholecystectomy in patients who underwent surgery for gallstone ileus. Methods: This was a retrospective review of the clinical history of patients who underwent surgery for gallstone ileus between December 1992 and December 2018 and follow-up until October 2020. Data regarding the surgical intervention, location of the obstruction, and surgical procedure performed were collected, as well as complications in relation to biliary pathology in the postoperative period. Results: Twenty-five patients underwent surgery for gallstone ileus. In all patients, except one, the site of the obstruction was identified. The mean age of the patients was 72 (standard deviation [SD] 13.3) years, with a female predominance (18: 7). The patients presented symptoms, on average, 2.9 (1-7) days before going to the emergency room; the primary symptoms were vomiting associated with abdominal pain and constipation (56\%). Fifty-six percent of patients were diagnosed preoperatively by imaging tests. In $72 \%$ of patients, an enterolithotomy was performed alone without any other intervention on the gallbladder or bile duct. Eighty-three percent of the patients did not present any cholecystobiliary complications during the entire follow-up period, and urgent or delayed cholecystectomy was not performed after the acute episode. Conclusions: Gall-
\end{abstract}

stone ileus is a rare entity, and there are no randomized studies that support a preferred treatment. If surgical intervention is required, enterotomy for stone extraction is a safe and effective technique, and in our experience, urgent or delayed cholecystectomy is not necessary.

(C) 2021 The Author(s)

Published by S. Karger AG, Basel

\section{Introduction}

Gallstone ileus is a rare complication of cholelithiasis, and it is described as the presence of a mechanical biliary obstruction by impaction of one or more large gallstones within the gastrointestinal tract. The stone passes to the digestive tract through a fistula, the most frequent type being located between the gallbladder and the duodenum [1], with the distal ileum being the place where the obstruction is most often located [2]. The mortality rate associated with this pathology ranges from 12 to $27 \%$, and the morbidity rate reaches $50 \%$, generally due to patient age and associated pathologies [3]. It represents $0.3-0.5 \%$ of the complications of gallbladder disease, and only $50 \%$ of patients have a previous history of biliary pathology [4].

Gallstone ileus is a mechanical obstruction of the gastrointestinal tract; therefore, the site where the stone is fixed will produce different clinical scenarios [5]. Barnard syndrome occurs when stones obstruct the ileocaecal valve $[4,6]$. Bouveret syndrome is caused by gastric outlet
(C) 2021 The Author(s)

Published by S. Karger AG, Basel

This is an Open Access article licensed under the Creative Common Attribution-NonCommercial-4.0 International License (CC BY-NC) (http://www.karger.com/Services/OpenAccessLicense), applicable to the online version of the article only. Usage and distribution for commercial purposes requires written permission.
Correspondence to:

FRANCISCA GARCIA-MORENO NISA, francisca.garciam@uah.es 
obstruction due to duodenal obstruction and appears in only $3 \%$ of patients $[2,7,8]$. Less than $5 \%$ of patients have obstruction at the level of the colon [9]. The clinical symptoms vary depending on the location of the obstruction; the diagnosis should be suspected in an older patient who presents with Mordor triad (history of gallstones, signs of acute cholecystitis, and sudden appearance of intestinal obstruction [4]). Finally, a chronic presentation is also possible, known as Karewsky syndrome, where the patient develops recurrent episodes of pain due to the passage of gallstones through the intestine, together with asymptomatic periods, leading to the development of complete obstruction in several stages $[4,10]$.

The diagnosis is a challenge because the symptoms are not unique to this pathology. Surgical treatment is performed as a result of a preoperative diagnosis or because of intraoperative findings, with enterotomy alone or combined with another procedure as common approaches. Currently, other therapeutic options have emerged, such as endoscopy, which may play a role in Bouveret syndrome [11]. We conducted a review of experience in our hospital to analyse the forms of presentation, the period of onset of symptoms, and especially the need for cholecystectomy in the second stage or the usefulness of endoscopic treatment.

\section{Materials and Methods}

A retrospective review of the clinical history of patients who underwent surgery for gallstone ileus in our centre between December 1992 and December 2018 and with follow-up until October 2020 was performed. The following variables were collected: age, sex, comorbidities, ASA score, history of previous biliary pathology, type and duration of symptoms, pre- and postoperative hospital stay, and postoperative complications.

All patients received an abdominal X-ray, identifying intestinal obstructions, pneumobilia, and the presence of lithiasis in atypical locations. Other tests, such as CT, abdominal ultrasound, and endoscopy, were also conducted.

Finally, data were collected regarding the surgical intervention, location of the obstruction, and surgical procedure performed, as well as complications related to postoperative biliary pathology (need for subsequent cholecystectomy, spontaneous resolution of the fistula, or symptoms related to biliary pathology in the subsequent months). Microsoft Excel ${ }^{\circledR}$ was used for data collection and statistical analysis, that is, comparing means and standard deviations (SDs). The data are expressed as the mean and SD.

\section{Results}

In the period of time studied, 25 patients who underwent gallstone ileus intervention were identified. In all patients, except one, the site of the obstruction was identified (the patient presented with dilation of small bowel loops without the presence of a stone). The mean age of
Table 1. Associated comorbidities of the patients studied

\begin{tabular}{ll}
\hline Pathology & Patients, \% \\
\hline Diabetes mellitus & 28 \\
Kidney insufficiency & 8 \\
Arterial hypertension & 56 \\
COPD & 8 \\
History of neoplastic disease & 8 \\
Autoimmune disease & 4 \\
ACVA & 12 \\
Ischaemic heart disease & 16 \\
Heart arrhythmia & 12 \\
Deep vein thrombosis & 8 \\
\hline
\end{tabular}

Table 2. Mean analytical values observed in the patients studied

\begin{tabular}{lll}
\hline Parameter & Observed value & Reference value \\
\hline Leukocytes & 12,148 & $(4.5-11.0) 10^{3} / \mu \mathrm{L}$ \\
Haemoglobin & 14.7 & $(12-16) \mathrm{g} / \mathrm{dL}$ \\
Creatinine & 1.6 & $(0.55-1.2) \mathrm{mg} / \mathrm{dL}$ \\
Urea & 110 & $(18-46) \mathrm{mg} / \mathrm{dL}$ \\
Bilirubin & 1.2 & $(0.30-1.20) \mathrm{mg} / \mathrm{dL}$ \\
GGT & 26.6 & $<38 \mathrm{U} / \mathrm{L}$ \\
GPT & 59 & $(10-49) \mathrm{U} / \mathrm{L}$ \\
GOT & 27.3 & $<34 \mathrm{U} / \mathrm{L}$ \\
CRP & 50.23 & $<1 \mathrm{mg} / \mathrm{L}$ \\
Albumin & 3.5 & $(3.2-4.8) \mathrm{g} / \mathrm{dL}$ \\
\hline
\end{tabular}

the patients was 72 (SD 13.3) years, with a female predominance (18: 7). A total of $72 \%$ of patients did not undergo ASA classification because they were operated on urgently; among the others, $24 \%$ were ASA II-III. The most frequently found comorbidities are provided in Table 1 ( 5 of the 25 patients had no previous diseases). Forty percent of patients had a history of biliary pathology (1 biliary colic, 6 symptomatic cholelithiasis, 2 acute cholecystitis, and 1 cholecystitis with biliary fistula); in another $60 \%$, there were no analytical, radiological, or clinical data on the existence of biliary pathology.

The patients presented with clinical symptoms, on average, 2.9 (1-7) days before going to the emergency room. This symptomatology consisted mainly of vomiting associated with abdominal pain and constipation (56\%), followed by vomiting and abdominal pain in $32 \%$. Less frequently (8\%), patients consulted for fever, abdominal pain, and vomiting or vomiting without other associated symptoms (4\%). The average time since they appeared in the emergency department and underwent surgery was 3.16 days, with an SD of $5.36(0.23)$.

The most frequent analytical alterations were leucocytosis and elevated creatinine and CRP levels (Table 2). Fifty-six percent of the patients were diagnosed preoperatively using imaging tests; among the others, diagnosis 
Table 3. Percentage of patients with radiological findings in diagnostic tests

\begin{tabular}{ll}
\hline Radiological test & Findings (\%) \\
\hline $\begin{array}{l}\text { Plain abdominal X-ray } \\
\text { (25 patients) }\end{array}$ & Extravesicular lithiasis (8) \\
& Dilation of the small intestine (48) \\
& Pneumobilia + small intestine dilation (8) \\
& Rigler's triad: (4) \\
\hline Abdominal ultrasound & Pneumobilia (7.7) \\
(13 patients) & Dilation of the small intestine (7.7) \\
& Cholelithiasis (23) \\
& Gastric bloating (15) \\
& Pneumobilia + cholelithiasis (15) \\
& Pneumobilia + small intestine dilation (7.7) \\
& Pneumobilia + cholelithiasis + small intestine dilation + intraluminal lithiasis (7.7) \\
& Cholelithiasis + small intestine dilation \\
& No findings (7.7) \\
\hline Abdominal CT (15 patients) & Dilation of the small intestine (6.6) \\
& Gastric dilation (6.6) \\
& Bilioenteric fistula + gastric dilation (13.2) \\
& Pneumobilia + small intestine dilation + extravesicular lithiasis (suspicion of \\
& cholecystoduodenal fistula) (60) \\
& Pneumobilia + small intestine dilation (13.2) \\
\hline
\end{tabular}

occurred intra-surgery because the patients were operated on urgently for intestinal obstruction without extravesicular biliary lithiasis being identified in preoperative imaging tests. The tests performed and their findings are described in Table 3. In 6 patients, the diagnosis was made by simple X-ray; in 5, by X-ray associated with CT; and in 3, with X-ray and ultrasound.

Twenty percent of the patients underwent upper digestive endoscopy, finding lithiasis in the duodenum in 2 patients, gastric lithiasis in 1 , and gastric retention in 2 . Endoscopy was performed for therapeutic purposes in patients with duodenal lithiasis, but the approach was not effective; therefore, both patients required urgent surgical intervention. In one of them, gastrostomy was performed, and in the other enterolithotomy, cholecystectomy, and closure of the fistula were performed.

Regarding surgical intervention, only 1 case involved a laparoscopic approach and $4 \%$ of patients had preoperative septic shock. The ileum was the most frequent location of the obstruction (64\%); in 1 patient, obstructive lithiasis was not located during the intervention. A cholecystoduodenal fistula was identified in only 3 of the patients. The surgical procedures performed are described in Table 4. Obstructive lithiasis was not identified in one of the patients. This patient was followed for 14 months without developing biliary complications during this period.

One of the patients died in the postoperative period due to kidney failure that did not respond to treatment. Among the other patients, evolution was satisfactory, with a mean postoperative stay of 13 (4-45) days. Sixty
Table 4. Surgical procedures performed in the operated patients

\begin{tabular}{|c|c|}
\hline Technique & $\begin{array}{l}\text { Cases, } \\
n / \%\end{array}$ \\
\hline Enterolithotomy & $18 / 72$ \\
\hline Enterolithotomy + cholecystectomy + fistula suture & $1 / 4$ \\
\hline Intestinal resection & $2 / 8$ \\
\hline $\begin{array}{l}\text { Enterolithotomy + cholecystectomy + fistula suture + bile } \\
\text { duct revision + Kher tube }\end{array}$ & $2 / 8$ \\
\hline Gastrotomy & $1 / 4$ \\
\hline Exploratory laparotomy & $1 / 4$ \\
\hline
\end{tabular}

percent of patients presented postoperative complications, the most frequent being surgical wound infection (53\%). Cardiac (6.67\%) and kidney (13.3\%) complications and postoperative ileus (13\%) were also observed. The patients were clinically followed up at the consultation, the median follow-up was 24 months (0.312), and the median was worsened by asymmetric distribution of the data.

The postoperative biliary morbidity analysis indicated that 4 of the 25 patients underwent cholecystectomy; of the remaining 21 , only 1 required posterior cholecystectomy due to the development of acute cholecystitis in the late postoperative period ( $>30$ days). In addition, 3 patients developed choledocholithiasis (resolved by ERCP). However, $83 \%$ of patients did not present any cholecystobiliary complications during the entire follow-up period, and no urgent or scheduled cholecystectomy was performed after the episode of gallstone ileus. 


\section{Discussion/Conclusion}

Gallstone ileus is a rare cause of intestinal obstruction that occurs mainly in elderly patients. Although cases have been described in adolescents, [12] the majority of patients are elderly. In those older than 65 years, gallstone ileus represents $25 \%$ of intestinal obstructions of the small intestine not due to strangulation $[13,14]$.

This pathology occurs more frequently in women than in men, and some publications report a ratio of 16:1 [1416]. In our study, the majority were women 18:7, and the average age was 72 years.

This entity is a diagnostic challenge because its symptoms are not exclusive and may be present in other pathologies that produce obstruction of the small intestine (adhesions, internal hernias, tumours, volvulus, etc.). Therefore, a history of previous biliary pathology can help with the diagnosis. In our study, $40 \%$ of patients had a history of biliary pathology ( 1 biliary colic, 6 symptomatic cholelithiasis, 2 acute cholecystitis, and 1 cholecystitis with biliary fistula); in another $60 \%$, there were no analytical, radiological, or clinical data of the existence of biliary pathology. These findings coincide with those reported by Ploneda-Valencia et al. [5], that is, the presence of previous biliary symptoms was approximately $50 \%$.

In general, there is an average of 7 days from the onset of symptoms of intestinal obstruction and surgical treatment $[4,5]$. In our study, there was an average of 2.9 days from the onset of symptoms until the patients consulted the emergency department. The symptoms consisted of vomiting, abdominal pain, and constipation (58\%). These suggestive symptoms of intestinal obstruction are the most frequently observed symptoms in all published series $[14,17]$.

Only $31-48 \%$ of patients have a preoperative diagnosis $[14,16]$. Plain abdominal X-ray in decubitus and standing positions has a diagnostic sensitivity of $40-70 \%[18,19]$. Rigler's triad is diagnostic and consists of the presence of radiopaque stones (only present in $10 \%$ of cases), pneumobilia (Gotta-Mentschler sign), and distention of the intestinal loops. The presence of 2 of these 3 signs establishes the diagnosis $[4,5,10,20]$, although it should be borne in mind that pneumobilia is not pathognomonic of gallstone ileus and can occur in other pathologies or endoscopic procedures. The combined use of abdominal ultrasound plus conventional radiology increases the sensitivity to $74 \%$ [21]. Finally, CT with contrast is considered the diagnostic technique of choice, with a sensitivity above $90 \%[4,21,22]$.

Rigler's triad is very helpful, but in our study, it was only present in $4 \%$ of patients. However, $56 \%$ of patients had a preoperative diagnosis, mainly due to the greater performance of $\mathrm{CT}$ as a diagnostic test.
Surgical management remains the management of choice because with medical treatment, the mortality rate increases to $26.5 \%[6,23]$. Many patients have concomitant pathology and dehydration from vomiting [17], and the fundamental prognostic factor for choosing a surgical approach is the duration of intestinal obstruction [24]. Doko et al. [17] reported that $40 \%$ of patients have concomitant pathology and $13 \%$ have 2 or more comorbidities. In our case, this percentage was significantly higher because only 5 of the 25 patients did not present concomitant pathology, with the presence of 2 or more previous diseases being the most frequent.

In addition to the preoperative situation, intraoperative findings are relevant for deciding the surgical technique. The surgical technique/practice has not changed in these years. Since it acts only on the gallbladder, only in recent years has the laparoscopic approach been included. The patients included cover a long period of time, where the laparoscopic approach in our centre was not so widespread.

Enterolithotomy consists of the extraction of the stone through an enterotomy, without performing any other procedure on the gallbladder or the entero-biliary fistula (1-stage surgery). Its objective is to resolve intestinal obstruction without subjecting the patient to other procedures that prolong the operative time or increase morbidity. Reisner and Cohen [13] compared mortality in patients who underwent enterotomy alone and patients who underwent 1-stage surgery. The results showed a mortality rate of 11.7 for patients who underwent enterotomy alone versus $16.9 \%$ for patients who had 1-stage surgery. They concluded that enterolithotomy was the technique of choice in these cases, even assuming that the recurrence rate for gallstone ileus is approximately $5 \%$ and up to $85 \%$ in the first 6 months after resolution of the obstructive picture. At present, it is considered a good approach for patients with significant comorbidities, haemodynamic instability, or high-risk surgical dissection [14, 15]. Ravikumar et al. [21] and Williams et al. [6] agree that the limiting factor for performing surgery at one time is the haemodynamic status of the patient, considering that enterolithotomy is the procedure of choice in unstable patients.

The authors who prefer this procedure consider the risks of recurrence and of developing gallstone cancer to be low $(15-1 \%)[1,6]$. In our series, only 5 patients did not present previous diseases associated with the poor preoperative situation; therefore, the chosen technique was mostly (72\%) enterolithotomy alone. The patients were clinically followed up at the consultation, the median follow-up was 24 months (0.312), and the median was worsened by asymmetric distribution of the data (Reviewer 2 and Reviewer 3 ).

Two-stage surgery consists of enterolithotomy alone with interval cholecystectomy plus fistula repair. Approx- 
imately $10 \%$ of patients with enterotomy alone have recurrent biliary symptoms $[1,2]$; therefore, 2 -stage surgery has been recommended for young patients at risk of subsequent biliary complications and for patients with retained gallstones with a risk of recurrent gallstone ileus $[4,25,26]$. There is no consensus on the time period between the first surgery and the second stage of the procedure, ranging from 4 weeks to 6 months [13, 25, 27].

Finally, the use of therapeutic endoscopy in this pathology is still under development. Endoscopy is reserved for patients with Bouveret syndrome (upper endoscopy) or ileal or colonic obstruction (colonoscopy) [4, 28]. Colonoscopy removal of impacted gallstones should be attempted in cases of colonic ileus. Interventional endoscopy in high-risk patients is the best treatment option [4, 7, 29]. Regarding our experience, we performed therapeutic endoscopy in 2 patients with duodenal lithiasis, and the approach was ineffective; therefore, the patients required surgical intervention. The number of cases prevents us from drawing conclusions regarding the efficacy of endoscopy, although it seems evident that it can play an important role in patients with duodenal lithiasis and a very poor baseline clinical situation with high surgical risk and in patients with colonic biliary lithiasis.

\section{Conclusions}

It is difficult to draw conclusions from a retrospective study of a rare disease over such a long period of time. Therefore, the study presents limitations to be able to establish definitive conclusions, since in addition, it is not possible to carry out a statistical study.

Gallstone ileus is a condition in elder patients with associated comorbidities. It should be suspected in older patients with intestinal obstruction, and when suspicious, radiological Rigler's signs should be sought. It is a rare entity, and there are no randomized studies that support the most correct treatment. If surgical intervention is required, enterotomy for stone extraction is a safe and ef- fective technique, and in our experience, urgent or delayed cholecystectomy is not necessary. Simple enterolithotomy is the treatment of choice for gallstone ileus; only in very select cases should an emergency procedure be performed in addition to a cholecystectomy and closure of the bilio-enteric fistula.

\section{Statement of Ethics}

The research was conducted ethically in accordance with the World Medical Association Declaration of Helsinki. The studies have been approved by the Hospital Universitario Principe De Asturias Ethics Committee (0E 46/2020). For a retrospective study, written informed consent from participants was not required in accordance with national guidelines.

\section{Conflict of Interest Statement}

The authors have no conflicts of interest to declare.

\section{Funding Sources}

No funding sources received.

\section{Author Contributions}

All authors have made substantial contributions to the conception and design of the study, acquisition of data, analysis and interpretation of data, drafting the article or revising it critically for important intellectual content, and final approval of the version to be submitted. C.V., A.N.S., B.M., and F.M.M.: data collection; F.G.M.N.: study design, and analysis and interpretation of data; and M.D.A.: drafting the article.

\section{Data Availability Statement}

All data generated or analysed during this study are included in this article. Further enquiries can be directed to the corresponding author.

\section{References}

1 Nuño-Guzman CM, Arróniz-Jáuregui J, Moreno-Pérez PA, Chávez-Solis EA, EsparzaArias N, Hernández-González CI. Gallstone ileus: one-stage surgery in a patient with intermitent obstruction. World J Gastrointest Surg. 2010;2:172-6.

2 Martín-Pérez J, Delgado-Plasencia L, Bravo Gutiérrez A, Burillo-Putze G, Martínez-Riera A, Alarcó-Hernández A, et al. Gallstone ileus as a cause of acute abdomen. Importance of early diagnosis for surgical treatment. Cir Esp. 2013;91:485-9.

3 Alexiou K, Ioannidis A, Sikalias N, Konstantinidou E, Fotopoulos A, Karanikas I, et al.
Gallstone ileus: a case report and our clinic's experience. Surg Sci. 2014;5:10-4.

4 Beuran M, Ivanov I, Venter MD. Gallstone ileus-clinical and therapeutic aspects. J Med Life. 2010;3:365-71.

5 Ploneda-Valencia CF, Gallo-Morales M, Rinchon C, Navarro-Muñiz E, Bautista-López, de la Cerda-Trujillo LF, et al. El íleo biliar: una revisión de la literatura médica. Rev Gastroenterol México. 2017;82:248-54.

6 Williams NE, Gundara JS, Roser S, Samra JS. Disease spectrum and use of cholecystolithotomy in gallstone ileus transection. Hepatobiliary Pancreat Dis Int. 2012 Oct;11(5):553-7.
7 Watson RS, Folkers TE, Van Every MJ. A multidisciplinary approach to management of Bouveret syndrome. Clin Med Res. 2018;16:73-5.

8 Santos-Rancaño R, Talavera Esquizábal P, Torres García AJ. Síndrome de Bouveret. Emergencias. 2016;28:131.

9 Creedon L, Boyd-Carson H, Lund J. A curious case of cololithiasis. Ann R Coll Surg Engl. 2018;100:e188-90.

10 Rodriguez-Hermosa JI, Codina-Cazador A, Gironés-Vila J, Roig García J, figa Francesch M, Acero Fernández D. Ileo-biliar: resultados del análisis de una serie de 40 casos. Gastroenterol Hepatol. 2001;24:489-94. 
11 Ali IA, Mahmood S, Tierney WM. Bouveret syndrome: a tough nut or crack. VideoGIE. 2019;4:126-7.

12 Grassi R, Pinto A, Rossi E, Rossi G, Scaglione $\mathrm{M}$, Lassandro F, et al. Nine consecutive patients with gall stone ileus. Personal experience. Radiol Med. 1998;95:177-81.

13 Reisner RM, Cohen JR. Gallstone ileus: a review of 1,001 reported cases. Am Surg. 1994; 60:441-6.

14 Rodriguez-Sanjuan JC, Casado F, Fernández MJ, Morales D, Naranjo A. Cholecystectomy and fistula closure versus enterolithotomy alone in gallstone ileus. Br J Surg. 1997;84: 634-7.

15 Sciacca P, Benini B, Borrello M. Mechanical occlusion of the small intestines by gallstones. Our experience. Miner Chir. 1997;52:39-44.

16 Freitag M, Elsner I, Günl U, Albert W, Ludwing $\mathrm{K}$. Clinical and imaging aspects of gallstone ileus. Experiences with 108 individual observations. Chirurg. 1998;69:265-9.
17 Doko M, Zovak M, Kopljar M, Glavan E, Ljubicic N, Hochstädter $\mathrm{H}$. Comparison of surgical treatments of gallstone ileus: preliminary report. World J Surg. 2003;27:400-4.

18 Muthukumarasamy G, VenKata SP, Shaikh IA, Somani BK, Ravindran R. Gallstone ileus: surgical strategies and clinical outcome. J Dig Dis. 2008;9:156-61.

19 Collins A, Coughlin D, Mullen M. Gallstone ileus. J Emerg Med. 2013;44:e277-8.

20 Chou JW, Hsu CH, Liao KF, Lai HC, Cheng KS, Peng CY, et al. Gallstone ileus: report of two cases and review of the literature. World J Gastroenterol. 2007;13:1295-8.

21 Ravikumar R, Williams JG. The operative management of gallstone ileus. Ann R Coll Surg Engl. 2010;92:279-81.

22 Lassandro F, Gagliardi N, Scuderi M, Pinto A, Gatta G, Mazzeo R. Gallstone ileus analysis of radiological findings in 27 patients. Eur J Radiol. 2004;50:23-9.

23 Webb LH, Ott MM, Gunter OL. Once bitten, twice incised: recurrent gallstone ileus. Am J Surg. 2010;200:e72-4.
24 Hayes N, Saha S. Recurrent gallstone ileus. Clin Med Res. 2012;10:236-9.

25 Berliner SD, Burson LC. One-stage repair for cholecyst-duodenal fistula and gallstone ileus. Arch Surg. 1965;90(2):313.

26 Shioi Y, Kawamura S, KannoNishinari KY, Nishinari Y, Ikeda K, Noro A, et al. A case of gallstone ileus displaying spontaneous closure of cholecystoduodenal fistula after enterolithotomy. Int J Surg Case Rep. 2012;3: 12-5.

27 Raf L, Spangen L. Gallstoneileus. Acta Chir Scand. 1971;137:665-75.

28 Kako S, Iwaya Y, Nagaya T, Umemura T. Successful treatment of gallstone ileus with double-balloon enteroscopy-assisted electrohydraulic lithotripsy. Dig Liver Dis. 2020 Jul 16; S1590-8658(20):30331-5.

29 Zielinski MD, Ferreira LE, Baron TH. Successful endoscopic treatment of colonic gallstone ileus using electrohydraulic lithotripsy. World J Gastroenterol. 2010;16:1533-6. 